\title{
Semiclassical treatment of fusion processes in collisions of weakly bound nuclei
}

\author{
L.F. Canto, R. Donangelo \\ Instituto de Física, Universidade Federal do Rio de Janeiro, \\ C.P. 68528, 21941-972 Rio de Janeiro, Brazil \\ H.D. Marta \\ Instituto de Física, Facultad de Ingeniería, \\ C.C. 30, C.P. 11000 Montevideo, Uruguay
}

\begin{abstract}
We describe a semiclassical treatment of nuclear fusion reactions involving weakly bound nuclei. In this treatment, the complete fusion probabilities are approximated by products of two factors: a tunneling probability and the probability that the system is in its ground state at the strong absorption radius. We investigate the validity of the method in a schematic two-channel application, where the channels in the continuum are represented by a single resonant state. Comparisons with full coupled-channels calculations are performed. The agreement between semiclassical and quantal calculations is quite good, suggesting that the procedure may be extended to more sophisticated discretizations of the continuum.
\end{abstract}

PACS numbers: 25.60.-t, 25.60.Pj, 24.10.-i

Keywords: Unstable beams, Fusion reactions, Projectile breakup 


\section{INTRODUCTION}

The effects of channel coupling in fusion reactions induced by weakly bound projectiles have attracted great interest over the last decade [1]. Some theoretical studies predict strong influence of the breakup channel over the complete fusion (CF) cross section 2, 3, 4, 5, 6, 4]. One of the reasons for this interest is the growing amount of experimental data on these processes [8, 9, 10, 11, 12, 13, 14], which are important both for the study of astrophysical processes as well as for the production of superheavy nuclei.

The appropriate theoretical tool to handle this problem is the coupled-channels method. However, its implementation becomes very complicated for the breakup channel, which is specially important in the case of weakly bound nuclei, since it involves an infinite number of states in the continuum. For practical purposes, it becomes necessary to approximate the continuum by a finite set of states, as in the Continuum Discretized Coupled-Channels method (CDCC) 15]. This procedure has been extended to the case of fusion reactions in refs. [5, 6, 7]. Recently, a semiclassical alternative based on the classical trajectory approximation of Alder and Winther (AW) [16] has been proposed [17]. This approximation was used to calculate breakup cross sections and the results were compared with those of the CDCC method. The agreement between these calculations was very good. Since this semiclassical version of the CDCC method is much simpler, it may be a very useful tool to calculate cross sections for other channels in reactions with weakly bound nuclei.

Although the AW method has been extensively used for several nuclear reaction processes, only very recently it was applied to the estimate of the fusion cross section [18]. For this application it was considered a simplified two bound channels problem for which the fusion cross section obtained with the AW method was compared with results of a quantal coupled-channels calculation. In spite of the large simplification in the problem, the agreement between these two calculations at above-barrier energies was very good. However, the semiclassical method severely underestimated the fusion cross section at sub-barrier energies. In the present work, we also consider two channels, but instead of being both bound, one of them represents a resonance in the continuum. We have assessed the validity of the semiclassical model in this case through comparisons with results of the corresponding full quantum mechanics coupled channels calculations. We have shown in particular that the good agreement with quantum mechanical calculations can be extended to sub-barrier 
energies through the analytical continuation of the time.

The paper is organized as follows: in section \we review the semiclassical treatment of nuclear reactions and give its extension to fusion reactions. In section III], we discuss the application of this treatment to the schematic two-channel model of ref. [18], and show that it severely underestimates the fusion cross section at sub-barrier energies. Then, in section IV] we develop a generalization of the semiclassical model which is accurate at sub-barrier energies and takes into account the width of the effective channel. The conclusions and discussion of further work are given in the last section.

\section{SEMICLASSICAL DESCRIPTION OF FUSION}

As this work is devoted to reactions induced by weakly bound projectiles, the variables employed to describe the collision are the projectile-target separation vector, $\mathbf{r}$, and the relevant intrinsic degrees of freedom of the projectile, $\xi$. For simplicity, we neglect the internal structure of the target. The Hamiltonian is then given by

$$
h=h_{0}(\xi)+V(\mathbf{r}, \xi)
$$

where $h_{0}(\xi)$ is the intrinsic Hamiltonian and $V(\mathbf{r}, \xi)$ represents the projectile-target interaction. The eigenvectors of $h_{0}(\xi)$ are given by the equation

$$
h_{0}\left|\phi_{\alpha}\right\rangle=\varepsilon_{\alpha}\left|\phi_{\alpha}\right\rangle
$$

The AW method [16] is implemented in two-steps. First, one employs classical mechanics for the time evolution of the variable $\mathbf{r}$. The ensuing trajectory depends on the collision energy, $E$, and the angular momentum, $\hbar l$. In its original version, an energy symmetrized Rutherford trajectory $\mathbf{r}_{l}(t)$ was used. In our case, the trajectory is the solution of the classical equations of motion with the potential $V(\mathbf{r})=\left\langle\phi_{0}|V(\mathbf{r}, \xi)| \phi_{0}\right\rangle$, where $\left|\phi_{0}\right\rangle$ is the ground state of the projectile. In this way, the coupling interaction becomes a time-dependent interaction in the $\xi$-space, $V_{l}(\xi, t) \equiv V\left(\mathbf{r}_{l}(t), \xi\right)$. The second step consists of treating the dynamics in the intrinsic space as a time-dependent quantum mechanics problem. Expanding the wave function in the basis of intrinsic eigenstates,

$$
\psi(\xi, t)=\sum_{\alpha} a_{\alpha}(l, t) \phi_{\alpha}(\xi) e^{-i \varepsilon_{\alpha} t / \hbar}
$$


and inserting this expansion into the Schrödinger equation for $\psi(\xi, t)$, one obtains the AW equations

$$
i \hbar \dot{a}_{\alpha}(l, t)=\sum_{\beta}\left\langle\phi_{\alpha}\left|V_{l}(\xi, t)\right| \phi_{\beta}\right\rangle e^{i\left(\varepsilon_{\alpha}-\varepsilon_{\beta}\right) t / \hbar} a_{\beta}(l, t) .
$$

These equations are solved with the initial conditions $a_{\alpha}(l, t \rightarrow-\infty)=\delta_{\alpha 0}$, which means that before the collision $(t \rightarrow-\infty)$ the projectile was in its ground state. The final population of channel $\alpha$ in a collision with angular momentum $l$ is $P_{l}^{(\alpha)}=\left|a_{\alpha}(l, t \rightarrow+\infty)\right|^{2}$ and the angle-integrated cross section is

$$
\sigma_{\alpha}=\frac{\pi}{k^{2}} \sum_{l}(2 l+1) P_{l}^{(\alpha)}
$$

To extend this method to fusion reactions, we start with the quantum mechanical calculation of the fusion cross section in a coupled channel problem. For simplicity, we assume that all channels are bound and have spin zero. The fusion cross section is a sum of contributions from each channel. Carrying out partial-wave expansions we get

$$
\sigma_{F}=\sum_{\alpha}\left[\frac{\pi}{k^{2}} \sum_{l}(2 l+1) P_{l}^{F}(\alpha)\right],
$$

with

$$
P_{l}^{F}(\alpha)=\frac{4 k}{E} \int d r\left|u_{\alpha l}\left(k_{\alpha}, r\right)\right|^{2} W_{\alpha}^{F}(r)
$$

Above, $u_{\alpha l}\left(k_{\alpha}, r\right)$ represents the radial wave function for the $l^{t h}$-partial-wave in channel $\alpha$ and $W_{\alpha}^{F}$ is the absolute value of the imaginary part of the optical potential associated to fusion in that channel.

To use the AW method to evaluate the fusion cross section, we make the approximation

$$
P_{l}^{F}(\alpha) \simeq \bar{P}_{l}^{(\alpha)} T_{l}^{(\alpha)}\left(E_{\alpha}\right)
$$

In eq.(8), $\bar{P}_{l}^{(\alpha)}$ is the probability that the system is in channel $\alpha$ at the point of closest approach on the classical trajectory, and $T_{l}^{(\alpha)}\left(E_{\alpha}\right)$ is the probability that a particle with energy $E_{\alpha}=E-\varepsilon_{\alpha}$ and reduced mass $\mu=M_{P} M_{T} /\left(M_{P}+M_{T}\right)$, where $M_{P}, M_{T}$ are respectively the masses of the projectile and target, tunnels through the potential barrier in channel $\alpha$.

We now proceed to study the CF cross sections in reactions induced by weakly bound projectiles. For simplicity, we assume that the GS is the only bound state of the projectile and that the breakup process produces only two fragments, $F_{1}$ and $F_{2}$. In this way, the labels 
$\alpha=0$ and $\alpha \neq 0$ correspond respectively to the GS and the breakup states represented by two unbound fragments. Neglecting any sequential contribution, the CF can only arise from the elastic channel. In this way, the cross section $\sigma_{C F}$ can be obtained from eq.(91), dropping contributions from $\alpha \neq 0$. That is,

$$
\sigma_{C F}=\frac{\pi}{k^{2}} \sum_{l}(2 l+1) P_{l}^{\text {Surv }} T_{l}^{(0)}(E),
$$

where

$$
P_{l}^{\text {Surv }} \equiv \bar{P}_{l}^{(0)}=\left|a_{0}\left(l, t_{c a}\right)\right|^{2}
$$

is usually called survival (to breakup) probability.

\section{COMPARISON WITH COUPLED-CHANNELS CALCULATIONS}

The accuracy of the semiclassical fusion cross section has recently been checked in a schematic two-channel calculation for the scattering of ${ }^{6} \mathrm{He}$ projectiles on a ${ }^{238} \mathrm{U}$ target at above-barrier energies [18]. The weakly bound ${ }^{6} \mathrm{He}$ nucleus dissociates into ${ }^{4} \mathrm{He}$ and two neutrons, with threshold energy $B=0.975 \mathrm{MeV}$. The elastic channel is strongly coupled to the breakup channel and the influence of this coupling on the fusion cross section is very important. In this calculation, the breakup channel was represented by a single effective state [19]. For simplicity, the effective channel was treated as a bound state but it was assumed to contribute only to incomplete fusion. Furthermore, the ingoing wave boundary condition is used in all these calculations. The CF cross section is therefore given by eq. (9). In ref. [18] the threshold energy was neglected and the same potential barrier was used for both channels, i.e. Wood-Saxon shapes for the real and imaginary potentials, with the parameters: $V_{0}=-50 \mathrm{MeV}, r_{0 r}=1.25 \mathrm{fm}, a_{r}=0.65 \mathrm{fm}, W_{0}=-50 \mathrm{MeV}, r_{0 i}=1.0 \mathrm{fm}$ and $a_{i}=0.65 \mathrm{fm}$. This calculation adopted a form factor with the radial dependence of the electric dipole coupling and the strength was chosen arbitrarily, so that the coupling modified appreciably the cross section predicted by the one dimension barrier penetration model. The results (figure 1 of ref. [18]) are shown here in figure 1 (a). Above the Coulomb barrier, the semiclassically calculated cross section is in very good agreement with the one obtained by the coupled-channels method. However, the situation is much worse at energies below the Coulomb barrier. This is illustrated in figure 1 (b), where the results of (a) are plotted on a logarithmic scale. As it usually occurs, the total fusion cross section at 

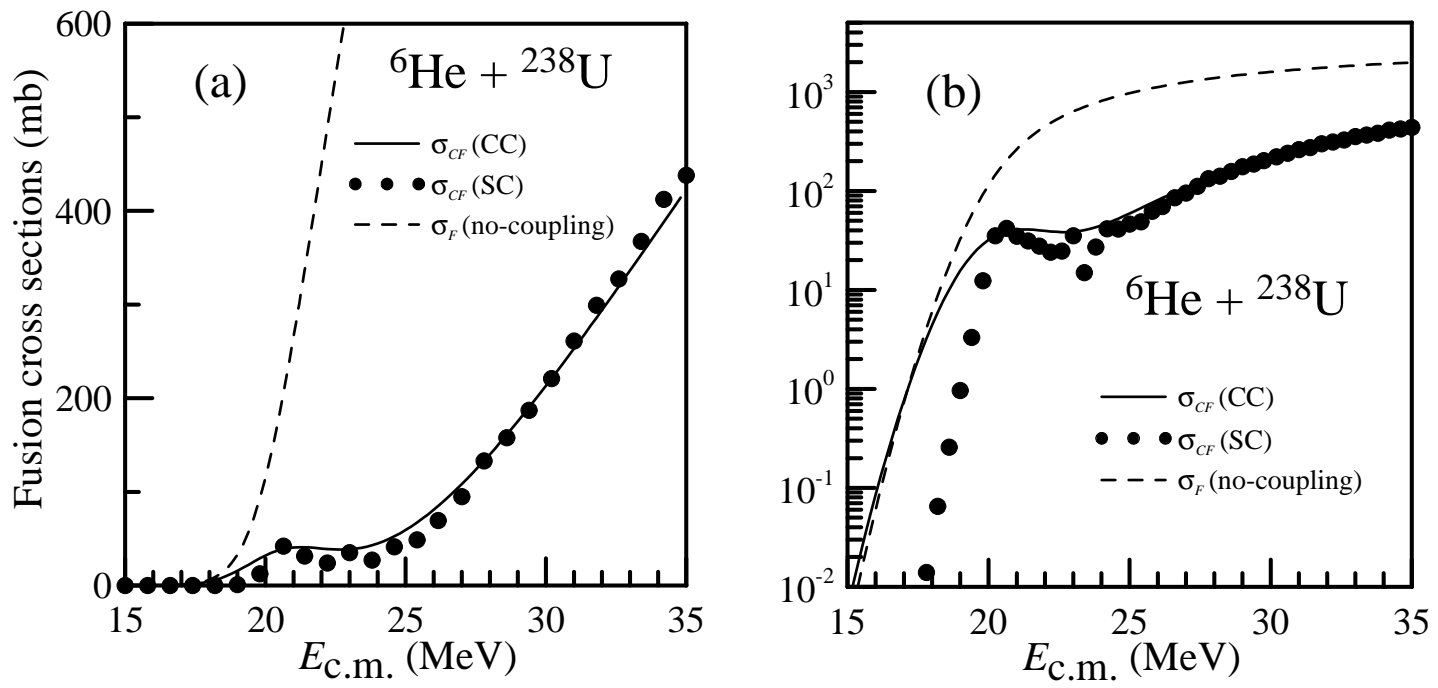

FIG. 1: Complete fusion cross section as calculated quantum mechanically (full line) compared with that of the Alder and Winther calculation (full dots) for the two channel case with $B=0$. (See text for details). For comparison, the fusion cross section calculated by quantum mechanics in the no coupling case is also shown (dashed line).

sub-barrier energies is enhanced in the $\mathrm{CC}$ calculation. The contribution from $\mathrm{CF}$ alone (solid line) is slightly larger than the fusion cross section in the no-coupling limit (dashed line). It is clear that the semiclassical calculation does not reproduce this effect, since its $\mathrm{CF}$ cross section is much lower in this energy range. This deficiency is due to the fact that in the semiclassical procedure described in section I the coupling is not considered inside the barrier region, because there is no classical trajectory connecting points inside this region. Therefore, the effective barrier lowering ocurring in the coupled-channels calculation cannot be reproduced. The semiclassical calculation shows also some instability for energies close to the barrier top $\left(V_{B} \simeq 20 \mathrm{MeV}\right)$. This last behavior results from orbiting effects in the classical trajectories.

\section{THE IMPROVED SEMICLASSICAL TWO-CHANNEL MODEL}

To improve the semiclassical model at sub-barrier energies, we resort to the analytical continuation method, which consists of introducing the imaginary part of the time variable 


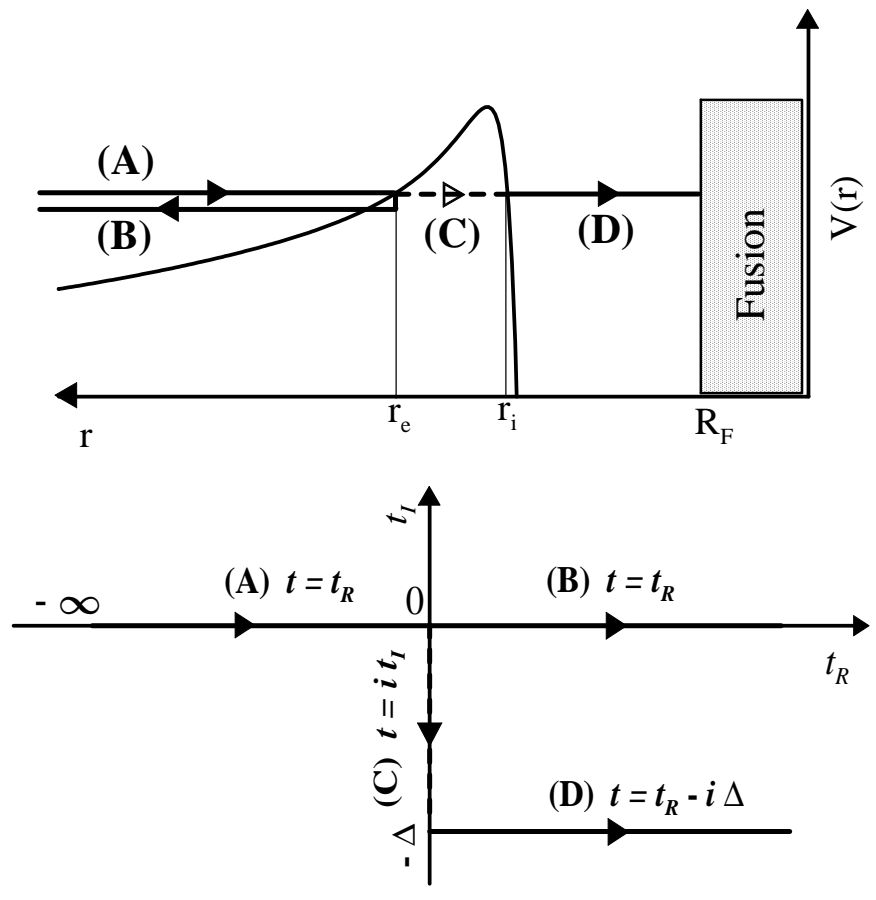

FIG. 2: Analytical continuation of the time variable. The upper panel shows the branches of the classical trajectory and the lower panel the evolution on the complex time plane.

to obtain a classical trajectory in the sub-barrier region [20]. This procedure is illustrated in figure 2, where the time scale is chosen such that $t=0$ at the external turning point, $r_{e}$. Along the incident branch of the trajectory $(\mathrm{A})$, the time develops on the real axis as the system approaches the barrier. At $r=r_{e}$, the trajectory splits into two parts: the reflected branch (B) and the classically forbidden transmission branch (C). On the former, which is not relevant to the fusion process, the time remains real. Along the branch (C), the real part of $t$ remains equal to zero while its imaginary part develops on the negative part of the imaginary axis, until this trajectory reaches the exit point $r_{i}$, at $t=-i \Delta$. This trajectory is then continued into the internal classically allowed region (D), towards the strong absorption radius, $R_{F}$, where fusion occurs. Over this branch, the real part of $t$ grows whereas its imaginary part keeps the value $t_{I}=-\Delta$.

We used the analytical continuation of $t$ in the calculation of the classical trajectories and generalized the AW equations (4) accordingly. In figure 3, CF cross sections of different calculations at sub-barrier energies are compared. The open and the full circles are respectively 


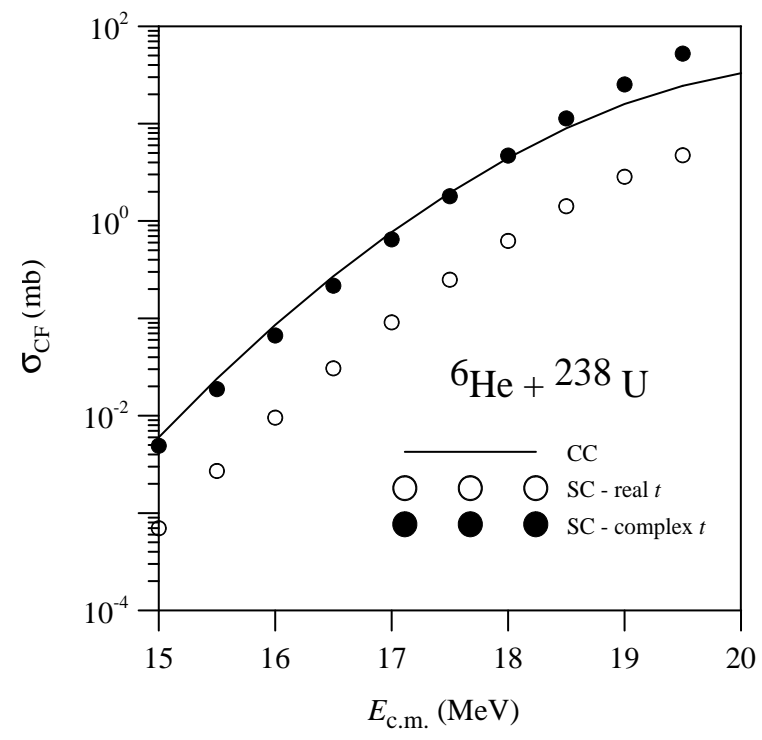

FIG. 3: Complete fusion cross section obtained with the calculations discussed in the text.

results from semiclassical calculations with real and complex times. The solid line represents results of coupled channel calculations. It is clear that the analytical continuation of the time variable improves substantially the accuracy of the semiclassical method. Now, their results agree very well with the ones of the quantum mechanical calculation, except for the last energy point, which is close to the barrier top. In this case, the cross section is strongly influenced by the orbiting effect in the classical trajectory. The system remains a very long time near the barrier radius, where the coupling is very strong. In this way, the elastic channel recovers the amplitude lost to the excited channel along the trajectory and the CF cross section becomes very large. This is a limitation of the semiclassical method. However, the situation tends do be much better in a realistic treatment of the continuum, with many channels and continuum-continuum coupling, owing to the irreversibility of the breakup process. The same occurs in the two-level model if the effective channel is treated as a doorway with finite width. This will be shown below.

To further improve the semiclassical calculation, we take into account the reaction $Q$ value. We assume that the excitation energy of the effective channel is the breakup threshold, $B$. The next step is to simulate the irreversible nature of the breakup process, identified in the CDCC calculations of Diaz-Torres and Thompson [6] by an effective channel with complex energy. To this end we use the fact that an exponentially decaying state with mean 


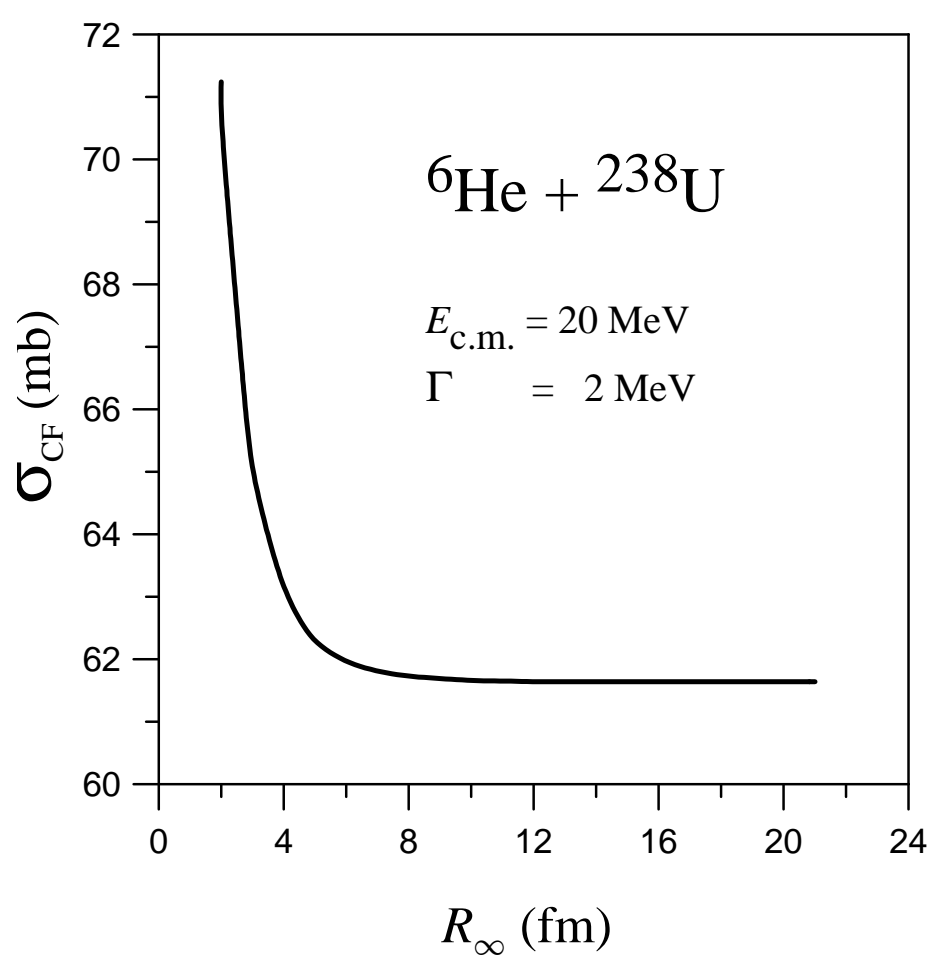

FIG. 4: Quantum calculation of the complete fusion cross section as a function of the cut-off radius of the potential $-i \Gamma / 2$. For details see the text.

life $\tau=\hbar / \Gamma$, can be obtained through the inclusion of a constant imaginary potential equal to $-i \Gamma / 2$ in the system Hamiltonian. This procedure, however, requires some care. The solution of the AW equations does not present difficulties since the population of the resonant state is vanishingly small as $t \rightarrow-\infty$. The numerical solution of the coupled-channel equations, however, requires attention. To handle this situation one should switch-off the $-i \Gamma / 2$ imaginary potential at some distance $R_{\infty}$ much larger than the range of the potentials, and then match the radial wave functions with their asymptotic forms. To illustrate this procedure, we show in figure 4 the $\mathrm{CF}$ cross section obtained through the solution of the coupled channel equations as a function of the cut-off radius. For this example, we considered the collision energy $E_{c . m} .=20 \mathrm{MeV} \simeq V_{B}$ and the typical width $\Gamma=2 \mathrm{MeV}$. The figure indicates that the result does not depend on the cut-off radius, provided that it is large enough $\left(R_{\infty} \gtrsim 20 \mathrm{fm}\right)$. 

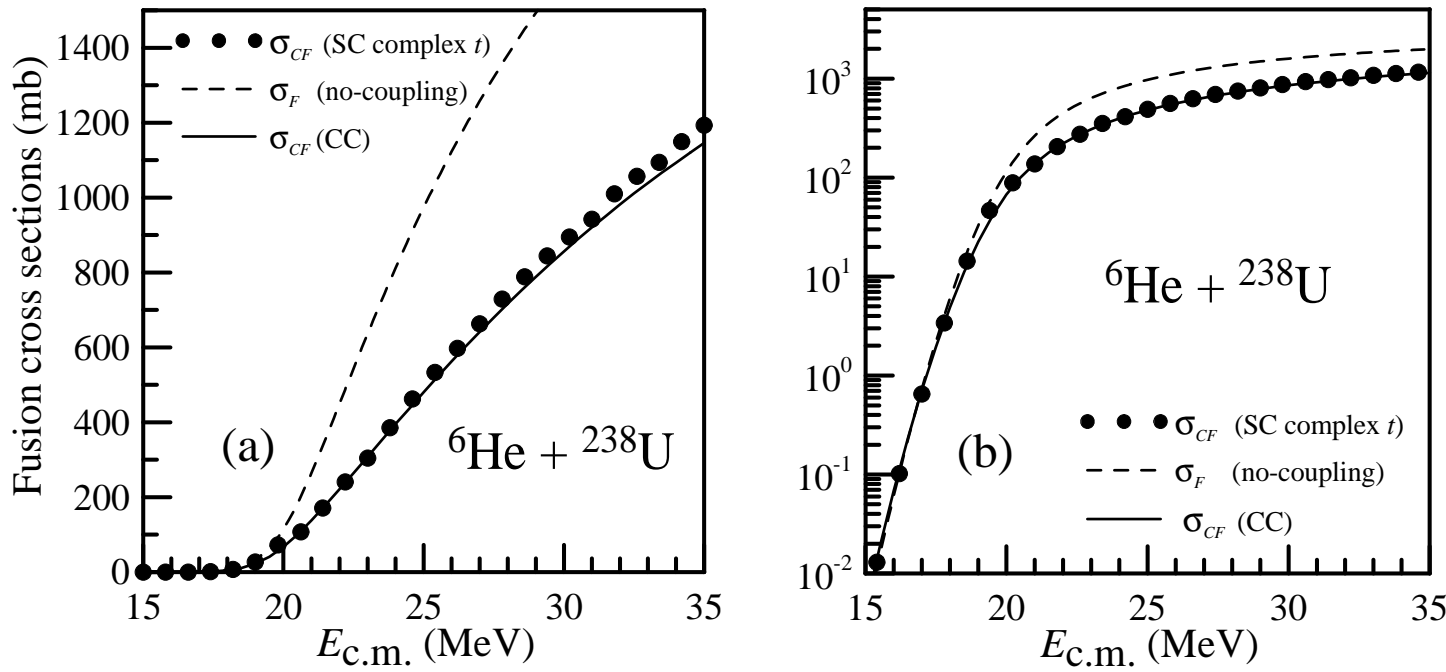

FIG. 5: Complete fusion cross section as calculated quantum mechanically (full line) compared with that of the present work (full circles), including the analytic continuation in the classically forbidden regions, for the two channel case with $B=0.975 \mathrm{MeV}$ and $\Gamma=2 \mathrm{MeV}$.

Let us now consider the CF cross sections obtained with the above discussed procedures. The results of the improved semiclassical calculation (solid circles) are shown in figure 5 , in comparison with results of the CC method (solid line) and in the no-coupling limit. In order to exhibit the details above and below the barrier, the cross sections are represented on a linear (a) and on a logarithmic (b) scale. Firstly, one observes that the suppression of the CF cross section at above-barrier energies is less pronounced than in figure 1. The reason for this difference is that here we are taking into account the breakup threshold energy, $B=0.975 \mathrm{MeV}$, while the calculations of figure 1 were performed in the sudden limit $(B=0)$. Nevertheless, the suppression of the CF cross section remains quite. Comparing the semiclassical estimate for $\sigma_{C F}$ with the CC values, we conclude that the improved semiclassical model leads to accurate results, above and below the Coulomb barrier.

\section{CONCLUSIONS}

We have illustrated how to extend the semiclassical method of Alder and Winther to the case of fusion reactions. Comparison of these calculations with full quantum ones for the 
case of only two channels, the elastic and an effective one, shows a good agreement between them. Below the barrier we have used the analytic continuation of the trajectory in the classically forbidden region, and we have shown it essential to describe correctly the process.

The fluctuations observed in the semiclassically calculated cross sections, in the case in which the effective channel has an infinite lifetime (zero width), may be associated to orbiting processes. However, when the resonance width is nonzero, the fluctuations vanish and the semiclassical calculations are in very good agreement with the full quantum ones. Thus it appears that fusion processes in weakly bound systems in which the elastic channel is coupled to the continuum are amenable to be treated by means of the extensions of the Alder and Winther's method presented here.

The extension of this semiclassical method to less schematic treatments of the continuum is presently under way [21]. In it the continuum is represented by a discrete set of channels, as in [6]; therefore the relative motion between the breakup fragments is more accurately described. In this way, detailed information on the positions and momenta of the projectile fragments at their creation point would be available. This would allow to study the collision of the fragments with the target, and therefore to estimate the incomplete fusion cross section as well as the sequential fusion contribution to the CF cross section. An estimate of this last process is not possible in the standard CDCC description of fusion [6], so semiclassical calculations may furnish a way to solve this problem. It should be remarked that a semiclassical study along these lines has already been implemented for calculations of the breakup cross section [17] and the results were in very good agreement with the ones obtained with CDCC calculations.

We acknowledge useful discussions with M.S. Hussein and P.R. Silveira Gomes. This work was supported in part by MCT/FINEP/CNPq(PRONEX) under contract no. 41.96.0886.00, PROSUL and FAPERJ (Brazil), and from PEDECIBA and CSIC (Uruguay).

[1] C.A. Bertulani, L.F. Canto and M.S. Hussein, Phys. Rep. 226, 281 (1993); C.A. Bertulani, M.S. Hussein and G. Münzenberg, "Physics of Radioactive Beams" (Nova Science, New York, 2001); M. S. Hussein, L.F. Canto and R. Donangelo, Nucl.Phys. A722, 321c (2003). 
[2] M.S. Hussein, M.P. Pato, L.F. Canto and R. Donangelo, Phys. Rev. C46, 377 (1992); C47, 2398 (1993).

[3] N. Takigawa, M. Kuratani and H. Sagawa, Phys. Rev. C47, R2470 (1993).

[4] C.H. Dasso and A. Vitturi, Phys. Rev. C50, R12 (1994).

[5] K. Hagino, A. Vitturi, C.H. Dasso and S.M. Lenzi, Phys. Rev. C61, 037602 (2000).

[6] A. Diaz-Torres and I.J. Thompson, Phys. Rev. C65, 024606 (2002).

[7] A. Diaz-Torres, I.J. Thompson and C. Beck, Phys. Rev. C68, 044607 (2003).

[8] M. Dasgupta et al., Phys. Rev. C66, 041602R (2002); M.Dasgupta et al., Phys. Rev. Letters 82, 1395 (1999).

[9] C. Signorini et al., Eur. Phys. J. A5, 7 (1999).

[10] C. Signorini et al., Eur. Phys. J. A13, 129 (2002).

[11] J.J. Kolata et al., Phys. Rev. Lett. 81, 4580 (1998).

[12] M. Trotta et al., Phys. Rev. Lett. 84, 2342 (2000).

[13] N. Alamanos et al., Phys. Rev. C65, 054606 (2002).

[14] K.E. Rehm et al., Phys. Rev. Lett. 81, 3341 (1998).

[15] F.M. Nunes and I.J. Thompson, Phys. Rev. C57, R2818 (1998); F.M. Nunes and I.J. Thompson, Phys. Rev. C59, 2652 (1999).

[16] K. Alder and A. Winther, Electromagnetic Excitations (North-Holland, Amsterdam, 1975).

[17] H.D. Marta, L.F. Canto, R. Donangelo and P. Lotti, Phys. Rev. C66, 024605 (2002).

[18] M.S. Hussein, L.F. Canto and R. Donangelo, invited talk at the International Symposium "A New Era of Nuclear Structure Physics", Nov. 19-22, 2003, Niigata, Japan, World Scientific, 2004. Preprint nucl-th/0403070.

[19] W.H.Z. Cárdenas et al., Phys. Rev. C68, 054614 (2003).

[20] S. Levit and U. Smilansky, in Proceedings of the Winter College on Fundamental Nuclear Physics, World Scientific, 1984.

[21] L.F. Canto, R. Donangelo and H.D. Marta, in preparation. 